\title{
Laserterapia diodo para estomatitis aftosa recurrente: reporte de un caso
}

\section{Diode lasertherapy for recurrent aphthous stomatitis: a case report}

\author{
Myriam Amparo Pulido-Rozo (iD) ${ }^{1}$, Xiomara Zilena Serpa-Romero (iD) ${ }^{2}$, Jorge Homero Wilches-Visbal (D) ${ }^{3}$ \\ 1. Universidad de Cartagena. Cartagena, Colombia. Correo: mpulidor@unicartagena.edu.co - https://orcid.org/0000-0003-1944-1618 \\ 2. Universidad del Magdalena. Santa Marta, Colombia. Correo: xserpa@unimagdalena.edu.co - https://orcid.org/0000-0002-8012-4067 \\ 3. Universidad del Magdalena. Santa Marta, Colombia. Correo: jwilches@unimagdalena.edu.co - https://orcid.org/0000-0003-3649-5079
}

Tipología: reporte de caso clínico

Para citar este artículo: Pulido-Rozo MA, Serpa-Romero XZ, Wilches-Visbal JH. Laserterapia diodo para estomatitis aftosa recurrente: reporte de un caso. Duazary. 2021 septiembre; 18(3 número especial): 114-120. Doi: https://doi.org/10.21676/2389783X.4266

Palabras

clave: láser diodo; equipo Wiser; estomatitis aftosa; úlcera; potencia.

\section{RESUMEN}

La estomatitis aftosa recurrente es una enfermedad oral caracterizada por la aparición de úlceras dolorosas en la mucosa labial o yugal. Su etiología es desconocida; suele afectar a personas menores de 30 años; y puede repetir durante meses o años. Antinflamatorios, glucocorticoides, antibióticos, entre otros, son tradicionalmente indicados para el manejo de las lesiones. Debido a los efectos colaterales de estos tratamientos, se ha sugerido la terapia láser. El objetivo del reporte fue establecer si el láser diodo de alta potencia desenfocado es efectivo contra la estomatitis aftosa de una paciente de 30 años, que padecía de úlceras bucales desde los 10 años, sin éxito con los tratamientos tradicionales. Las lesiones se irradiaron con $1 \mathrm{~W}$ de láser diodo, modo continuo con fibra de $400 \mu \mathrm{m}$ seguido de onda plana y pieza disfocal angulada en lengua, labio interno y carrillos. Asimismo, se aplicó laserpuntura en zona extraoral, para un total de 10 sesiones. Se observó reducción completa del dolor al tercer día de irradiación. Después de 1 año de seguimiento no se evidenció recurrencia. Se concluye que el láser diodo mostró mejores resultados que los anteriores tratamientos al aliviar rápidamente el dolor, evitar la recidiva y mejorar la autoestima de la paciente.

\section{ABSTRACT}

Keywords: Diode laser; Wiser equipment; Aphthous stomatitis; Ulcer; Power.
Recurrent aphthous stomatitis is an oral disease characterized by the appearance of painful ulcers on the labial or jugal mucosa. Its etiology is unknown; it usually affects people under 30 years old; being able to repeat for months or years. Anti-inflammatories, glucocorticoids, antibiotics, among others, are traditionally indicated for the management of lesions. Due to the side effects of these treatments, laser therapy has been suggested. The objective of the report was to establish whether the defocused highpower diode laser is effective against aphthous stomatitis in a 30-year-old patient, who suffered from mouth ulcers since the age of 10 , without success with traditional treatments. The lesions were irradiated with $1 \mathrm{~W}$ diode laser, continuous mode with $400 \mu \mathrm{m}$ fiber followed by plane wave and angled disfocal piece on the tongue, inner lip and cheeks. Likewise, laser puncture was applied in the extraoral area, for a total of 10 sessions. Complete pain reduction was observed on the third day of irradiation. After 1 year of follow-up, no recurrence was evidenced. It is concluded that the diode laser showed better results than the previous treatments by quickly relieving pain, preventing recurrence, and improving the patient's self-esteem. 


\section{INTRODUCCIÓN}

La estomatitis aftosa recurrente (EAR) es la afección benigna y dolorosa más frecuente de la mucosa oral ${ }^{1-3}$. Se caracteriza por múltiples úlceras pequeñas de forma ovoide, color gris amarillento y borde eritematoso ${ }^{1,4}$. Por lo general, una sensación de ardor precede de 2 a 48 h, la aparición de las úlceras. Estas se localizan en las mucosas labial o yugal y rara vez en encías ${ }^{1}$. Dado que otras enfermedades como el herpes bucal, la colitis ulcerosa o el virus de inmunodeficiencia adquirida (VIH) también causan úlceras, compete al odontólogo hacer el diagnóstico diferencial con la estomatitis aftosa. ${ }^{1}$ La EAR se clasifica en menor, mayor y herpetiforme. La menor, conocida como úlceras aftosas leves, representa el $80 \%$ de los casos y en individuos con edades de 5 a 19 años. La mayor o periadenitis mucosa necrótica recurrente, se presenta entre el $10-15 \%$ de los casos en adolescentes entre los 10 y 19 años. La hepertiforme, consiste en brotes periódicos de hasta 100 úlceras de 2-3 mm de diámetro. Este tipo de EAR es más común en mujeres de 20 a 29 años ${ }^{1,4}$.

La EAR es considerada una enfermedad multifactorial asociada a factores genéticos, inmunológicos, hormonales y al estrés ${ }^{2}$. Para establecer el pronóstico de la EAR y el efecto terapéutico de las técnicas empleadas, se utiliza un índice compuesto por la actividad de la úlcera, el dolor y la discapacidad funcional derivada. La actividad de la úlcera (AU) es 0 si no existe; 1 si hay una o más. El dolor se expresa en una escala visual analógica (EVA), donde el paciente establece su intensidad en una línea de 0 a $10 \mathrm{~cm}$. Marcación en $0 \mathrm{~cm}$ : ausencia de dolor; 1-3: leve-moderado; 4-6: moderado-grave; 6-9: intenso y 10: insoportable ${ }^{5}$. La discapacidad se refleja en la escala Likert, 0: en ningún caso la úlcera limita la deglución, el habla o la masticación; 1: poco tiempo; 2: algunas veces; 3 : la mayoría de las veces y 4 : todo el tiempo ${ }^{4}$. Su abordaje depende de la gravedad de los síntomas, el diámetro, número de úlceras y la frecuencia de aparición. Para lesiones pequeñas ocasionales, pueden usarse agentes antinflamatorios no esteroideos como el zilactin, orabase, mezclados o no con benzocaína, o diclofenaco; en aquellas más frecuentes o graves, se aplican glucocorticoides tópicos para disminuir el tiempo de cicatrización; en lesiones grandes, inyecciones de esteroides en las lesiones son indicadas. ${ }^{1}$ Antibióticos, como la tetraciclina tópica, han sido utilizados con baja tasa de éxito. ${ }^{1}$ Otras medidas tienen que ver con cambio en la alimentación, mejorar la higiene oral y suministro de suplementos vitamínicos ${ }^{2}$. Dado que los efectos secundarios de estos medicamentos se han asociado al abandono del tratamiento, desde hace unos años se ha propuesto la terapia láser como alternativa ${ }^{3}$.

El láser se define como un haz intenso generado por la emisión estimulada de radiación de una fuente luminosa ${ }^{6}$. La emisión estimulada consiste en excitar un electrón a un nivel elevado de energía por absorción de un fotón para después promover su retorno a su estado estable (o metaestable) por choque con otro fotón. Ambos fotones (el que estimula como el que promueve el retorno) tienen la misma energía, frecuencia y dirección ${ }^{7}$. Los parámetros dosimétricos que caracterizan al haz e inciden en la absorción tisular son: energía (en J) y potencia (en $W$ ), densidad de energía o dosis/fluencia $\left(\mathrm{J} / \mathrm{cm}^{2}\right)$, densidad de potencia o intensidad $\left(\mathrm{W} / \mathrm{cm}^{2}\right)$, duración del pulso (en s), longitud de onda (en $\mathrm{nm}$ ), distancia al tejido (en $\mathrm{mm}$ ), diámetro de la fibra (en $\mu \mathrm{m}$ ) y tiempo de irradiación (en s). El modo de aplicación puede ser continuo ( $\mathrm{CW}$ en inglés) o pulsado ( $\mathrm{PW}$ en inglés) ${ }^{8}$. Reportar tales parámetros es imprescindible para realizar validaciones y comparaciones entre diferentes tratamientos y equipos láser ${ }^{9}$.

Los láseres se clasifican según el medio de activación $^{6}$; longitud de onda, potencia y aplicación clínica $^{8}$. Los láseres de alta potencia $(>500 \mathrm{~mW})$ se usan en cirugías, mientras los de media (250-500 $\mathrm{mW}$ ) y baja potencia ( $<250 \mathrm{~mW}$ ) para bioestimular, regenerar tejidos, acelerar la cicatrización y paliar el dolor ${ }^{10}$. Dos de sus principales ventajas son la alta direccionalidad al foco de la lesión y la poca afectación a las células sanas circundantes ${ }^{11}$. La respuesta del tejido depende de su estado, la condición del sistema inmune, la técnica de 
tratamiento, la longitud de onda y la intensidad, las tres últimas definidas por el odontólogo, según las especificaciones del equipo ${ }^{11}$. El parámetro dosimétrico más relevante para inducir reacciones fotoquímicas es la intensidad ${ }^{11}$.

El láser diodo, de estado sólido ${ }^{8}$ y con franja de emisión en el infrarrojo cercano $(700-1200 \mu \mathrm{m})^{11}$, es muy empleado en odontología ${ }^{10}$, especialmente en endodoncia ${ }^{12}$. La razón es que el coeficiente de absorción de la hemoglobina y la melanina es alto en esa franja ${ }^{13}$, haciendo que su energía sea fuertemente capturada en los tejidos blandos pero poco en dientes y huesos ${ }^{14}$.

Láseres diodo de alta potencia simulan a los de baja cuando el haz es desenfocando (o disfocal) ${ }^{14}$, al incrementar el área de irradiación ${ }^{15,16}$. Con esto, se aprovecha la bondad de ambos, en un solo equipo: corte de tejidos (alta potencia) en modo contacto (con fibra óptica) y analgesia/reparación (baja potencia) para modo no-contacto (sin fibra o alejada) $)^{8,14,17}$. Además, en modo superpulsado (pulsos $\leq 100$ ms: casi continuo) no es necesario aplicar anestesia $^{14,18}$.

En este trabajo, se describe un reporte de caso de EAR en paciente femenina de 30 años, utilizando láser diodo de un equipo Wiser (Doctor Smile) ${ }^{19}$.

\section{REPORTE CASO CLÍNICO}

Paciente de sexo femenino de 30 años con lesiones aftosas múltiples en lengua, carrillos, mucosas labiales, pilares amigdalinos, con 3 días de evolución y recurrencia de 4 veces al año en promedio, desde los 10 años, acude a la Facultad de Odontología, Universidad de Cartagena, en 2015, para valoración. La paciente es cartagenera, madre cabeza de hogar, sometida a altos niveles de estrés asociado a las responsabilidades del hogar, trabajo, estudios profesionales nocturnos y antecedentes sistémicos de cólicos menstruales y estrés.

Desde 2 años antes (2013) manifiesta estar sufriendo lesiones ulcerosas más frecuentes, numerosas, extremadamente dolorosas (AU: 1; EVA:10; Likert: 4) e incapacitantes (Figura 1), impidiéndole hablar, comer y cepillarse los dientes.
Hasta entonces recibió corticoides, antibióticos orales, tópicos y sistémicos, anestésicos locales, antiinflamatorios $y$ orabase, sin resultados satisfactorios a mediano plazo.
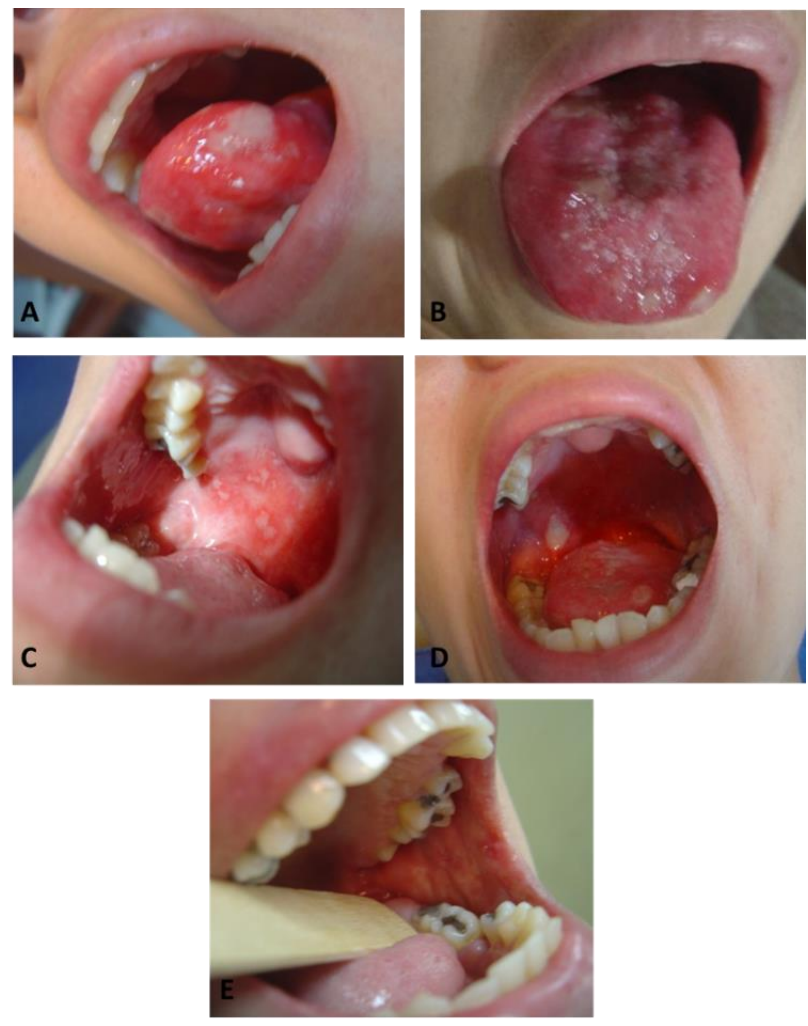

Figura 1. Lesiones multifocales en lengua (A y B); paladar blando (C y D) y carrillo (E).

Por ello, se decide realizar terapia láser diodo ( $1 \mathrm{~W}$; $\mathrm{CW}$; fibra de $400 \mu \mathrm{m}$ ), arrancando con 2 sesiones de descontaminación, en lengua y carrillo, con contacto intermitente en movimiento continuo durante $60 \mathrm{~s}$, aplicando previamente anestésico tópico (Figura $2 \mathrm{~A})$. Después de $60 \mathrm{~s}$, se continua con la pieza de mano disfocal angulada por $60 \mathrm{~s}$ a la misma potencia y a $0,5 \mathrm{~mm}$ de distancia de la mucosa labial anterior (Figura 2B). 

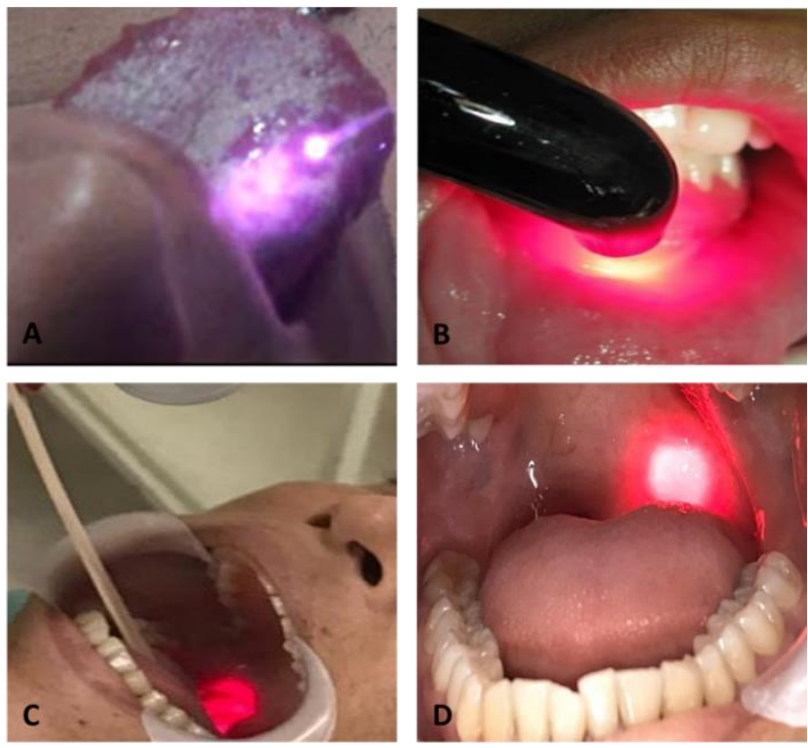

Figura 2. Irradiación láser con fibra de vidrio de $400 \mu \mathrm{m}$ para descontaminación $(A)$ y disfocal angulada en mucosa labial (B) con onda plana en paladar blando ( $C$ y D).

En los pilares amigdalinos y paladar blando se usó onda plana $\left(1 \mathrm{~W} / \mathrm{cm}^{2}\right.$ durante $60 \mathrm{~s}$ para dosis de $60 \mathrm{~J} / \mathrm{cm}^{2}$, en $\mathrm{CW}$ ) con movimientos pequeños (Figura $2 \mathrm{C}$-D). Se aplicó laserpuntura ${ }^{20}(0,7 \mathrm{~W}$ durante $0,5 \mathrm{~s}$, $\mathrm{CW}$ ) con pieza disfocal angulada durante $5 \mathrm{~s}$ en cada punto a 0,5 $\mathrm{mm}$ de distancia de la piel. Desde la tercera sesión solo se irradió con onda plana y angulada, para bioestimular y promover la cicatrización de las úlceras. Las terapias se ejecutaron diariamente, totalizando 10 sesiones. No hubo terapia coadyuvante a la de láser.

Los resultados arrojaron mejoría inmediata del dolor. A la tercera sesión, la EVA quedó en 0 , notándose recuperación absoluta en la funcionalidad de los sitios lesionados (Likert: 0, AU: 0; EVA: 0) a la décima sesión (Figura 3).

Se sugirió control cada tres meses. Después de 12 meses, no se vieron efectos colaterales ni recidivas. Desaparecieron la sialorrea y la halitosis. La mucosa oral recuperó su color, textura y humectación. Consecuentemente, el estado de ánimo de la paciente mejoró y, con ello, su autoestima y calidad de vida.
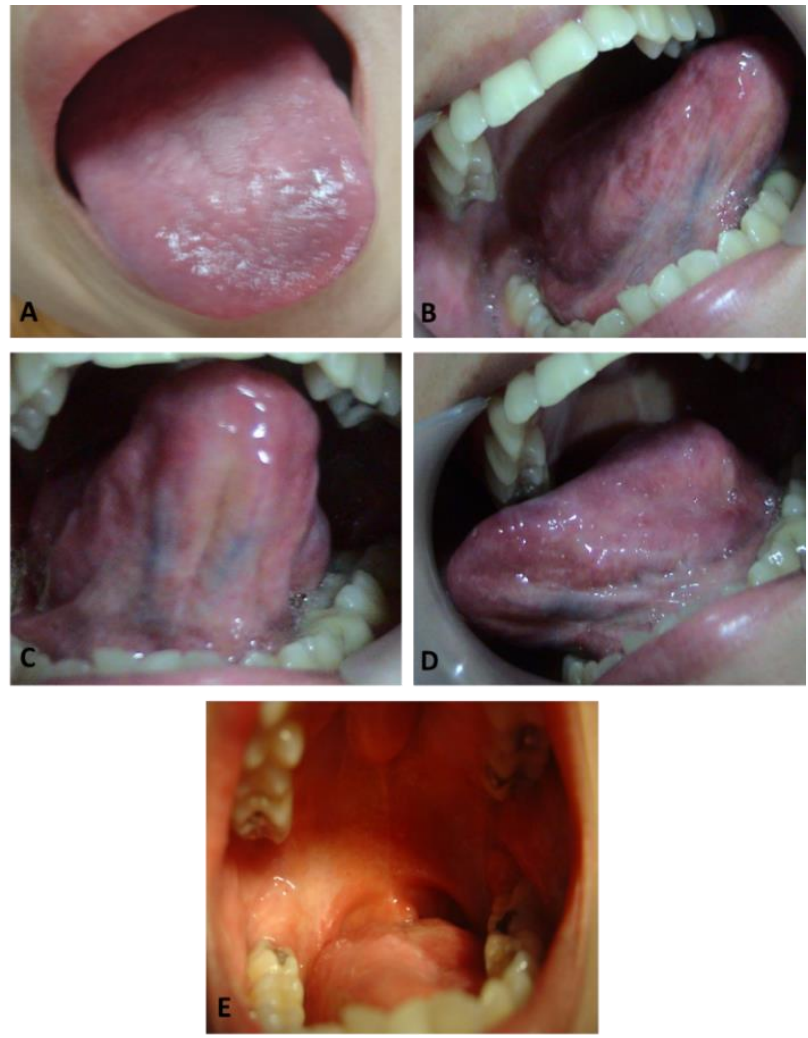

Figura 3. Cicatrización de las lesiones en lengua (A, B, C y $D)$ y orofaringe $(E)$, después de 10 sesiones.

\section{Declaración sobre aspectos éticos}

Los autores declaran que para este estudio se contó con el consentimiento informado del paciente sobre su participación, así como para la utilización de datos de su historia clínica y fotografías, sin comprometer su identidad. Los procedimientos se ajustaron a los principios éticos de la declaración de Helsinki y la resolución 8430 de 1993 del Ministerio de Salud de Colombia. Se resguardó la confidencialidad y custodia de la información acorde a la Ley Estatutaria 1581 de 2012.

\section{DISCUSIÓN}

La estomatitis aftosa recurrente es una enfermedad oral que afecta seriamente la calidad de vida del individuo. Desde años atrás se ha abordado exitosamente utilizando láser de baja potencia o de alta potencia en modo disfocal ${ }^{14,17,18,21,22}$. En Colombia existen pocos reportes sobre laserterapia para EAR, ninguno de ellos trabajando con equipo Wiser $^{23,24}$. 
Farista et al. ${ }^{16}$, en un paciente de 21 años con conglomerados de pequeñas úlceras en la lengua que causaban dolor al masticar, utilizaron laser diodo (DC Lase, $980 \mathrm{~nm}, 1 \mathrm{~W}$, modo desenfocado y continuo sin punta o no-contacto) a $10 \mathrm{~mm}$ de distancia de la lesión y acercándose progresivamente hasta $2 \mathrm{~mm}$ con movimientos circulares, en 3 ciclos de $20 \mathrm{~s}$ de duración con $10 \mathrm{~s}$ de intervalo entre cada uno. Luego de 3 días, las lesiones estaban parcialmente curadas sin dolor. La curación completa se vio 4 días después.

Misra et $a l^{18}$ emplearon laserterapia diodo (Ezlase, $0,1 \mathrm{~W}, 3 \mathrm{~J} / \mathrm{cm}^{2}, 940 \mathrm{~nm}$, modo pulsado y nocontacto) durante 1 min en cada úlcera de la mucosa labial de un paciente de 25 años con recurrencia de 3 veces en 1 mes. Mostraron que el paciente no experimentó dolor durante la sesión, ni ardor después de esta. Las úlceras cicatrizaron 4 días después de la aplicación; 2 meses después no vio recurrencia.

Hazeem et $a 1^{14}$ en un trabajo realizado sobre 32 personas, con al menos 2 úlceras, tratadas con láser diodo (Ezlase, 4W, $940 \mathrm{~nm}$, modo pulsado de $1 \mu \mathrm{s} /$ pulso y no-contacto, 1 sesión), encontraron que 3 días después, no había cicatriz y que las lesiones cedieron completamente a los $7 \pm 2$ días.

Anand et $a l^{21}$, emplearon laserterapia diodo en dos pacientes, uno de ellos con historial de recurrencia de 7 años a pesar de haber sido tratado con anestésicos tópicos y esteroides. La terapia fue aplicada con láser desenfocado a una distancia inicial de 5-8 mm y acercándose con movimientos de redondeados en la lesión hasta 2-3 mm. Ejecutaron 3 ciclos el mismo día en modo continuo (940 nm), con potencia de 0,6 W, incrementado $0,1 \mathrm{~W}$ sucesivamente en las dos sesiones siguientes, para igual tiempo de exposición de 30-45 s. Vieron mejoría después de 3-4 días, sin recurrencia durante 1 año de seguimiento.

Tal como sucedió en este trabajo, los antes descritos pudieron tratar efectivamente la EAR, sin complicaciones evidentes. Se destaca que, al año de seguimiento, no hubo recaídas. Se observa un uso variado de la potencia aplicada y tiempo de aplicación menor o igual a $1 \mathrm{~min}$. El estudio de Farista et $a l^{16}$, aplicó la misma potencia que el presente, para igual longitud de onda. En ambos estudios, el dolor cedió a los 3 días.

Aunque la causa del poder analgésico y restaurativo del láser no se conoce con certeza, parece estar relacionada con varios mecanismos, entre otros: aumento de la producción mitocondrial de adenosín trifosfato (ATP) y disminución en el consumo de oxígeno celular; incremento de serotonina y endorfinas; disminución de la síntesis de prostaglandinas; inducción de factores de crecimiento y citocinas que resulta en disminución de la inflamación $y$, consecuentemente, en la cicatrización ${ }^{14}$. Otras teorías al respecto tienen que ver con el bloqueo del potencial de acción de la neurona aferente primaria; la mejora en la microcirculación local y más flujo de oxígeno a células hipóxicas, incremento de la cantidad de analgésicos naturales como los opioides o reducción de la síntesis de bradicinina y prostaglandina $E 2^{18}$.

Se concluye que la terapia con láser diodo de equipo Wiser para EAR (980 nm, 1W y modo continuo) produjo un rápido alivio del dolor, cicatrización de las úlceras, no recidiva a mediano-largo plazo $\mathrm{y}$, consecuentemente, una mejora en la calidad de vida; mostrando así, mejores resultados que terapias tradicionales anteriormente empleadas en la paciente.

\section{DECLARACIÓN SOBRE CONFLICTO DE INTERÉS}

Los autores declaran la inexistencia de conflictos de intereses respecto a la ejecución y redacción del manuscrito.

\section{CONTRIBUCIÓN DE LOS AUTORES}

Primer autor: conceptualización, metodología y ejecución del procedimiento clínico.

Segundo autor: búsqueda bibliográfica y revisión. Tercer autor: búsqueda bibliográfica, redacción y revisión final.

\section{REFERENCIAS BIBLIOGRÁFICAS}

1. Akintoye SO, Greenberg MS. Recurrent Aphthous Stomatitis. Dent Clin North Am [Internet]. abril de 2014;58(2):281-97. Disponible en: 
https://linkinghub.elsevier.com/retrieve/pii/S0011 853213001316

2. Sánchez-Bernal J, Conejero C, Conejero R. Aftosis oral recidivante. Actas Dermosifiliogr [Internet]. julio de 2020;111(6):471-80. Disponible en: https://linkinghub.elsevier.com/retrieve/pii/S0001 731020300545

3. Khaleel Ahmed M, Jafer M, Nayeem M, Hussain Moafa I, Quadri MFA, Gopalaiah H, et al. Low-Level Laser Therapy and Topical Medications for Treating Aphthous Ulcers: A Systematic Review. J Multidiscip Healthc [Internet]. noviembre de 2020; 13:1595$605 . \quad$ Disponible en: https://www.dovepress.com/low-level-lasertherapy-and-topical-medications-for-treatingaphthous--peer-reviewed-article-JMDH

4. Preeti L, Magesh K, Rajkumar K, Karthik R. Recurrent aphthous stomatitis. J Oral Maxillofac Pathol [Internet]. 2011;15(3):252. Disponible en: http://www.jomfp.in/text.asp?2011/15/3/252/866 69

5. Vicente Herrero MT, Delgado Bueno S, Bandrés Moyá F, Ramírez Iñiguez de la Torre MV, Capdevila García L. Valoración del dolor. Revisión Comparativa de Escalas y Cuestionarios. Rev la Soc Española del Dolor [Internet]. 2018; Disponible en: http://gestoreditorial.resed.es/DOI/PDF/ArticuloD Ol_3632.pdf

6. Luke AM, Mathew S, Altawash MM, Madan BM. Lasers: A Review With Their Applications in Oral Medicine. J Lasers Med Sci [Internet]. 1 de octubre de 2019;10(4):324-9. Disponible en: http://journals.sbmu.ac.ir/jlms/article/view/23044

7. Bogdan Allemann I, Kaufman J. Laser Principles. En: Current Problems in Dermatology [Internet]. 2011. p. 7-23. Disponible en: https://www.karger.com/Article/FullText/328236

8. Olivi G, De Moor R, DiVito E. Lasers in Endodontics [Internet]. Olivi G, De Moor R, DiVito E, editores. Lasers in Endodontics: Scientific Background and Clinical Applications. Cham: Springer International Publishing; 2016. 297 p. Disponible en: http://link.springer.com/10.1007/978-3-31919327-4

9. Matys J. Energy and Power Density: A Key Factor in Lasers Studies. J Clin DIAGNOSTIC Res [Internet]. 2015;9(12):ZL01-2. Disponible en: http://jcdr.net/article_fulltext.asp?issn=0973709x\&year=2015\&volume=9\&issue=12\&page=ZL01 \&issn=0973-709x\&id=6955

10. Torre D G, Márquez P R, Pozos G. A de J, Garrocho R. A. Usos del láser terapéutico en Odontopediatría: Revisión de la literatura. Reporte de casos. Odovtos - Int J Dent Sci [Internet]. 2018;20(3):51-9. Disponible en: https://www.medigraphic.com/cgibin/new/resumen.cgi?IDARTICULO=82177

11. Asnaashari M, Safavi N. Application of Low level Lasers in Dentistry (Endodontic). J Lasers Med Sci [Internet]. 2013;4(2):57-66. Disponible en: https://www.ncbi.nlm.nih.gov/pmc/articles/PMC42 81979/

12. España-Tost AJ, Arnabat-Domínguez J, BeriniAytés L, Gay-Escoda C. Aplicaciones del láser en Odontología. RCOE [Internet]. octubre de 2004;9(5). Disponible en: http://scielo.isciii.es/scielo.php?script=sci_arttext\& pid=S1138-

$123 \times 2004000500002 \& \operatorname{lng}=e n \& \mathrm{nrm}=\mathrm{iso} \& \mathrm{t} \operatorname{lng}=e n$

13. Parker S. Laser: Tissue Interaction and Its Application in Clinical Dentistry. Int J Laser Dent [Internet]. diciembre de 2011;1(1):1-8. Disponible en: $\quad$ https://www.ijold.com/doi/10.5005/jpjournals-10022-1001

14. Hazeem MI, Rajab MS, Badeia RA. Treatment of Recurrent Aphthous Stomatitis with 940nm Diode Laser. Tikrit J Dent Sci [Internet]. 2013;1:77-82. Disponible en: https://www.researchgate.net/publication/261063 282_Treatment_of_Recurrent_Aphthous_Stomatiti s_with_940nm_Diode_Laser

15. Cem B. Biomedical Optics and Lasers. En: A Roadmap of Biomedical Engineers and Milestones [Internet]. InTech; 2012. Disponible en: 
http://www.intechopen.com/books/a-roadmap-ofbiomedical-engineers-and-milestones/biomedicaloptics-and-lasers

16. Farista S, Kalakonda B, Ahmed AS. Effectiveness of $980 \mathrm{~nm}$ Diode Laser Therapy on Recurrent Aphthous Stomatitis. Int J Laser Dent [Internet]. diciembre de 2014;4(3):83-6. Disponible en: https://www.ijold.com/doi/10.5005/jp-journals10022-1062

17. Munna K, Alam N. Low Level Laser Therapy for Non-Invasive Dental applications: A Review. Int J Eng Res [Internet]. 6 de agosto de 2020;V9(07). Disponible en: https://www.ijert.org/low-levellaser-therapy-for-non-invasive-dental-applicationsa-review

18. Misra N, Maiti D, Misra P, Singh AK. $940 \mathrm{~nm}$ diode laser therapy in management of recurrent apthous ulcer. Case Reports [Internet]. 17 de abril de 2013;1:1-3. Disponible en: https://casereports.bmj.com/lookup/doi/10.1136/ bcr-2012-008489

19. Doctor Smile. Laser Wiser [Internet]. Lambda SpA. 2021 [citado 21 de abril de 2021]. p. 1. Disponible en: https://www.doctorsmile.com/wiser-laser-diodo/

20. Valiente Zaldívar CJ, Garrigó Andreu MI. Láser blando en puntos de acupuntura para el tratamiento de enfermedades bucales. Rev Cubana Estomatol [Internet]. 1997;34(1):5-10. Disponible en: scielo.sld.cu/scielo.php?script=sci_arttext $\&$ pid=S00 34-75071997000100001

21. Anand V, Gulati M, Govila V, Anand B. Low level laser therapy in the treatment of aphthous ulcer. Indian J Dent Res [Internet]. 2013;24(2):267. Disponible en: http://www.ijdr.in/text.asp?2013/24/2/267/11669 1

22. Vázquez Marrero Al, Pérez Suárez $M$ de la $C$, García Zaldívar ME. Eficacia del láser en el tratamiento de la estomatitis aftosa recurrente. $\mathrm{CCH}$, Correo cient Holguín [Internet]. 2019;23(1):281-7. Disponible en: http://scielo.sld.cu/scielo.php?script=sci_arttext\&p id=S1560-43812019000100281

23. Guzmán de Suárez B, Arias Rojas A. Manejo clínico de un paciente con estomatitis aftosa recurrente aplicando laser terapéutico. Rev Estomatol [Internet]. 1993;3(1):51-64. Disponible en:

https://bibliotecadigital.univalle.edu.co/bitstream/ handle/10893/2463/Manejo clinico.pdf?sequence=1\&isAllowed=y

24. Pulido Rozo M, Madera Anaya M, Tirado Amador L. Laser terapia en el manejo de aftas mayores: reporte de un caso. Acta odontol venez [Internet]. 2013;51(1). Disponible en: https://www.actaodontologica.com/ediciones/201 3/1/art-17/ 